\title{
Denoising of Mechanical Vibration Signals Using Quantum-Inspired Adaptive Wavelet Shrinkage
}

\author{
Yan-long Chen, ${ }^{1}$ Pei-lin Zhang, ${ }^{1}$ Bing Li, ${ }^{2}$ and Ding-hai Wu ${ }^{1}$ \\ ${ }^{1} 7$ th Department, Ordnance Engineering College, Shijiazhuang, China \\ ${ }^{2} 4$ th Department, Ordnance Engineering College, Shijiazhuang, China \\ Correspondence should be addressed to Yan-long Chen; chenyanlong110@163.com
}

Received 10 November 2013; Accepted 26 March 2014; Published 9 April 2014

Academic Editor: Valder Steffen Jr.

Copyright (c) 2014 Yan-long Chen et al. This is an open access article distributed under the Creative Commons Attribution License, which permits unrestricted use, distribution, and reproduction in any medium, provided the original work is properly cited.

\begin{abstract}
The potential application of a quantum-inspired adaptive wavelet shrinkage (QAWS) technique to mechanical vibration signals with a focus on noise reduction is studied in this paper. This quantum-inspired shrinkage algorithm combines three elements: an adaptive non-Gaussian statistical model of dual-tree complex wavelet transform (DTCWT) coefficients proposed to improve practicability of prior information, the quantum superposition introduced to describe the interscale dependencies of DTCWT coefficients, and the quantum-inspired probability of noise defined to shrink wavelet coefficients in a Bayesian framework. By combining all these elements, this signal processing scheme incorporating the DTCWT with quantum theory can both reduce noise and preserve signal details. A practical vibration signal measured from a power-shift steering transmission is utilized to evaluate the denoising ability of QAWS. Application results demonstrate the effectiveness of the proposed method. Moreover, it achieves better performance than hard and soft thresholding.
\end{abstract}

\section{Introduction}

Safety of a mechanical system is very important for industry. The study of fault feature detection in machinery has thus received considerable attentions during the past decades. Among all detection methods, the most popular tool is vibration-based analysis. However, for the studies about practical mechanical vibration signals, noise is an inevitable factor in the measured signals which always inhibits the extraction of true signal signatures for diagnosis. Therefore, noise depressing in mechanical time series is an important issue for accurate fault diagnosis.

Compared with conventional methods, as an effective analysis technique, wavelet transform is a frequently used tool for nonstationary signal processing in many fields. Various shrinkage strategies in the wavelet domain have been proposed for denoising. The most popular methods are VisuShrink, SureShrink, BayesShrink, and NeighShrink. Recently, several shrinkage functions have been modified for better noise reduction based on the above shrinkage approaches [1-8].
Chesneau et al. [1] presented a stein block thresholding algorithm for denoising $d$-dimensional data. Taylor et al. [2] denoised single-molecule fluorescence resonance energy trajectories using wavelet detail thresholding. The paper [3] described a new shrinkage methodology based on non-Gaussian statistical modelling for multimodal image denoising. Liu et al. [4] explored a suitable threshold in a complete solution space using particle swarm optimization. The work [5] developed a threshold-based wavelet denoising method for hydrological data modelling. Chen and Qian [6] proposed a new denoising method for hyperspectral data cubes. Wang et al. [7] developed a denoising algorithm incorporating DTCWT with NeighCoeff shrinkage. Shao et al. [8] diagnosed gear transmission system using wavelet threshold denoising based on EMD. All these wavelet shrinkage algorithms achieved better denoising performance in corresponding fields than conventional techniques.

The work of Wang et al. [7] indicates that the DTCWTbased denoising method for vibration signals is effective compared to those DWT-based and SGWT-based NeighCoeff shrinkage denoising methods. DTCWT reduces spectral 
aliasing for vibration signals and enjoys nearly shift invariance which are attractive properties favorable to both the surveillance and diagnosis of machinery. Owing to these advantages, DTCWT is employed to denoise mechanical vibration signals in our method.

It must be noted that the functionality of all the methods aforementioned is due to the thresholding rule, which determines the threshold value. There is thus potential to explore a novel thresholding rule to extend wavelet shrinkage. Quantum-inspired techniques based on quantum theory have been recently proposed and successfully applied to search unsorted data [9], solve various optimization problems [10-12], process signals [13, 14], despeckle images [15], and so forth. Experimental results show that using quantum mechanics effectively balances speed and effect.

With the inspiration of the basic principles of quantum signal processing (QSP) [16], a denoising method based on quantum theory and DTCWT using the quantum-inspired adaptive wavelet shrinkage (QAWS) is proposed to reduce noise in the machinery. There are two innovative aspects of the present work. One is that an adaptive non-Gaussian statistical model of DTCWT coefficients is proposed, and the other is that quantum theory is adopted to analyze the probability of noise considering interscale dependencies of wavelet coefficients in vibration signals. Denoising results of a signal collected from a mechanical power-shift steering transmission with inner race bearing fault demonstrate that the novel denoising approach yields the promising result than the soft and hard wavelet shrinkage technique.

The remainder of the paper is organized as follows. In Section 2, an adaptive wavelet coefficients statistic model is presented and a wavelet-domain Bayesian denoising processor is implemented to get a basic wavelet shrinkage function. In Section 3, using the concept of quantum theory, the quantum superposition is employed to compute the probability of noise while taking into account the dependencies between the DTCWT coefficients and their parents, and the basic wavelet shrinkage function in Section 2 updates to the QAWS. In Section 4, comparisons among the proposed QAWS technique, hard and soft thresholding introduced by Donoho and Johnstone [17], are implemented to denoise the vibration signal of a failure rolling element bearing in a mechanical power-shift steering transmission. Finally, the paper is concluded with a short summary in Section 5.

\section{Basic Shrinkage Function}

In this paper, a mechanical signal $y$ corrupted by white Gaussian noise $n$ with zero mean will be discussed, the signal is modelled as

$$
y=x+n
$$

where $x$ is a noise free signal. Random variables $x$ and $n$ are considered to be independent. We observe $y$ (a noisy signal) and attempt to estimate the noise free signal $x$ as accurately as possible. Unfortunately, it is difficult to estimate $x$ in time domain. Hence, we choose to estimate the wavelet coefficients of $x$ to solve this problemin wavelet domain. In the DTCWT domain, the problem can be expressed as

$$
Y=X+N
$$

where $Y=Y_{r}+i Y_{i}, X=X_{r}+i X_{i}$, and $N=N_{r}+i N_{i}$ are the complex wavelet coefficient of $y, x$, and $n$, respectively.

Our aim is to estimate $X$ from the noisy observation $Y$. To address this problem, the maximum a posteriori (MAP) estimator in a Bayesian framework will be used. The prior distribution of wavelet coefficients is necessary for the MAP estimator. A statistic model of DTCWT coefficients will be discussed in order to derive a basic wavelet coefficient shrinkage function.

It has been observed that wavelet coefficients of mechanical vibration signals have highly non-Gaussian statistics. The Bayesian theorem requires an appropriate prior; therefore, a new adaptive non-Gaussian statistical model of the DTCWT coefficient $X$ is proposed as the probability distribution function $(\mathrm{PDF})$

$$
p_{X}(X)=\frac{1}{2 \pi \sigma^{2}} \exp \left(-\frac{X_{r}^{2}+X_{i}^{2}}{2 \sigma^{2}} \exp (K)\right) .
$$

The imaginary and real parts of the DTCWT coefficients are distributed independently in our prior model. The motivation for constructing the statistical model is the availability of easily derived denoising functions. $K$ is an adaptive shape parameter for this model, which control the cusp and tail behaviours of the non-Gaussian statistical model. The smaller the shape parameter $K$ is, the heavier the tail of the corresponding density function becomes. The proposed PDF can provide a better fit to the wavelet coefficients than the PDF for wavelet coefficients in the Gaussian distribution method. This model is used to estimate $X$ by MAP estimator. As a result, $K$ also influences the denoising performance.

From the assumption on the noise, the distribution of $N$ is a zero mean Gaussian density with variance $\sigma_{n}^{2}$; that is,

$$
p_{N}(N)=\frac{1}{2 \pi \sigma_{n}^{2}} \exp \left(-\frac{N_{r}^{2}+N_{i}^{2}}{2 \sigma_{n}^{2}}\right) .
$$

The standard MAP estimation for $X$ given the noisy observation $Y$ is $\widehat{X}$

$$
\widehat{X}=\underset{X}{\arg \max } p_{X \mid Y}(X \mid Y) .
$$

Under the Bayesian framework, this equation can be written as

$$
\widehat{X}=\underset{X}{\arg \max }\left[p_{N}(N) \times p_{X}(X)\right] .
$$

Realizing the fact that computing the MAP estimator directly is difficult in this case, a logarithmic transformation is employed to convert (6) to a more tractable form

$$
\widehat{X}=\underset{X}{\arg \max }\left[\log \left(p_{N}(N)\right)+\log \left(p_{X}(X)\right)\right] .
$$


By using (3) and (4), (7) is represented as

$$
\widehat{X}=\underset{X}{\arg \max }\left[-\frac{(Y-X)^{2}}{2 \sigma_{n}^{2}}-\frac{X^{2}}{2 \sigma^{2}} \exp (K)\right] .
$$

Then, the MAP estimator of $X$ can be derived as

$$
\widehat{X}=\frac{\sigma^{2}}{\sigma^{2}+\sigma_{n}^{2} e^{K}} \times Y .
$$

Equation (9) is the basic shrinkage function in this paper. The shrinkage function contains the filter with the least estimated mean square error as a special case, corresponding to $K=0$. The special filter is written as follows:

$$
\widehat{X}=\frac{\sigma^{2}}{\sigma^{2}+\sigma_{n}^{2}} \times Y .
$$

The noise variance $\sigma_{n}^{2}$ is given by the real component of wavelet coefficient $Y$ at the finest scale of an original signal. Consider

$$
\widehat{\sigma_{n}^{2}}=\left(\frac{\operatorname{median}\left(\left|Y_{r}\right|\right)}{0.6745}\right)^{2}
$$

$\sigma^{2}$ is robustly estimated as follows:

$$
\widehat{\sigma^{2}}=\max \left(\frac{1}{M(s)} \sum_{m \in W(s, m)}|Y(s, m)|^{2}-\sigma_{n}^{2}, 0\right),
$$

where $|Y(s, m)|$ denotes the modulus of DTCWT coefficient $Y(s, m)$ at the $s$ th scale, $W(s, m)$ is a subwindow centered at wavelet coefficient $Y(s, m)$, and $M(s)$ denotes the size of $W(s, m)$. Here, the width of a subwindow can be expressed in terms of its scale parameter $s$

$$
M(s)=2^{d-s+1}-1
$$

where $d$ denotes the finest decomposition scale.

\section{Quantum-Inspired Adaptive Wavelet Shrinkage (QAWS)}

3.1. The Concept of Quantum Bit. In principle of quantum theory, the smallest unit of information is quantum bit (qubit). The state of a qubit can be described by a superposition of basic states $|0\rangle$ and $|1\rangle$ as follows [11]:

$$
|\phi\rangle=\alpha|0\rangle+\beta|1\rangle,
$$

where $|\phi\rangle,|0\rangle,|1\rangle$ is the quantum state, and $\alpha$ and $\beta$ denote the probability amplitudes of the corresponding states. In the representation, $|\alpha|^{2}$ and $|\beta|^{2}$ give the probability that the qubit will be in the " 0 " state and " 1 " state, respectively. The state of the qubit $|\phi\rangle$ varies as $\alpha$ and $\beta$ change. A qubit may be in the " 1 " state or in the " 0 " state or in any superposition of the two. Therefore, a qubit can represent all the states simultaneously in a data set. In quantum theory, a datum has a different way to be represented. We will show later that this would be more effective.

$\alpha$ and $\beta$ are complex number, and the following normalization condition is always satisfied:

$$
|\alpha|^{2}+|\beta|^{2}=1
$$

One should notice that the "spin up" and "spin down" orientations are not the sole interpretation of a quantum state. The "ground state" and the "excited state" of an atom, which represents $|0\rangle$ and $|1\rangle$, respectively, are also eligible [10]. Moreover, inspired by quantum mechanism, we can utilize quantum theory to analyze a mechanical vibration signal, while $|0\rangle$ specifies the state of noise and $|1\rangle$ specifies the state of true signal.

3.2. Quantum-Inspired Adaptive Wavelet Shrinkage (QAWS). The shape parameter $K$ is a key variate in the basic shrinkage function equation (9). It not only controls the shape of the presented PDF but also influences the denoising performance. Following Section 3.1, we focus on incorporating quantum theory into the shape parameter $K$ to update the basic shrinkage function to the quantum-inspired adaptive wavelet shrinkage (QAWS). The shape parameter $K$ is tuned by the quantum-inspired probability of noise. The QAWS characterizes interscale dependency between a coefficient and its parent and achieves a degree of noise reduction in the noisy mechanical signal.

The works of many authors have shown strong evidence that wavelet coefficients produced by true signal exhibit significant dependencies on subsequent locations. However, the coefficients caused by noise will decay rapidly along scales [18]. The magnitudes of noise coefficients have weaker correlation across the wavelet decomposition scales than those of true signal coefficients. Estimating a true signal by analyzing interscale correlated coefficients has been widely used in denoising algorithms. Therefore, in this paper, the correlation of wavelet coefficients across scales is combined to improve the noise-free coefficients estimation.

For any given wavelet coefficient, we consider interscale dependency between the child coefficient $(Y(s+1, m)$, finer scale) and corresponding parent coefficient $(Y(s, m)$, coarser scale) at the same position. Products of the coefficients and their parents are exploited and explored to measure the interscale dependencies of coefficients at the adjacent wavelet scales. At $m$ th position of the sth scale level, the product is written as

$$
C_{s m}=|Y(s+1, m)| \times|Y(s, m)| .
$$

Equation (16) is utilized in the high frequency subbands to distinguish between true signal and noise. Based on the fact that DTCWT coefficients of a mechanical vibration signal is a superposition of noise coefficients and true signal coefficients, the qubit is employed to describe $C_{s m}$. By using the basic principle of QSP [15], $C_{s m}$ can be formulated in a superposition state as follows:

$$
\left|C_{s m}\right\rangle=a|0\rangle+b|1\rangle
$$


where $|0\rangle$ denotes noise state and $|1\rangle$ denotes true signal state; the probability to have noise is $|a|^{2}$ and the probability to have true signal is $|b|^{2}$. In order to satisfy the relation of $|a|^{2}+|b|^{2}=$ 1 , the value of $C_{s m}$ is normalized as $N C_{s m} \in[0,1] . N C_{s m}$ also can reflect the interscale dependencies to some degree. Then, state $\left|C_{s m}\right\rangle$ is written as

$$
\left|C_{s m}\right\rangle=\cos \left(N C_{s m} \times \frac{\pi}{2}\right)|0\rangle+\sin \left(N C_{s m} \times \frac{\pi}{2}\right)|1\rangle .
$$

Now, $a=\cos \left(N C_{s m} \times \pi / 2\right)$ and $b=\sin \left(N C_{s m} \times\right.$ $\pi / 2)$, so the normalization condition $|a|^{2}+|b|^{2}=1$ is always guaranteed. The quantum-inspired probability of noise $N(s, m)$ in the sth level subband is equal to $\cos ^{2}\left(N C_{s m} \times\right.$ $\pi / 2)$, while the quantum-inspired probability of true signal $X(s, m)$ in the sth level subband is equal to $\sin ^{2}\left(N C_{s m} \times\right.$ $\pi / 2)$. At a certain location, if a wavelet coefficient exhibits significant dependency between the child and its parent, which means the true signal likely exists, $C_{s m}$ will be larger and $\sin ^{2}\left(N C_{s m} \times \pi / 2\right)$ will be larger as well. Contrariwise, if the noise is likely to exist, $C_{s m}$ will be less and $\cos ^{2}\left(N C_{s m} \times\right.$ $\pi / 2$ ) will be larger.

Note that the $K$ is allowed to vary between coefficients rather than being the same for all coefficients in a given subband in order to achieve better performance. Then, we determine the parameter $K$ adaptively by

$$
K=k_{0}+\cos ^{2}\left(N C_{s m} \times \frac{\pi}{2}\right),
$$

where $k_{0}$ is a tunable parameter determined by complex coefficients $Y$. From now on, (9) becomes the quantuminspired adaptive wavelet shrinkage (QAWS). The parameter $K$ can improve the versatility of the presented QAWS.

The value of $k_{0}$ can be estimated by applying the curve fitting technique to the histogram distribution of wavelet coefficient $Y . k_{0}$ in the $s$ th level is denoted by $k_{0 s}$. The mean square error (MSE) is chosen as the objective evaluation criteria. The less the MSE is, the more accurate the detail is. Consider

$$
k_{0 s}=\underset{k_{0 s}}{\arg \max } E\left(p_{T Y_{s}}\left(T Y_{s}\right)-p_{Y_{s}}\left(Y_{s}\right)\right)^{2},
$$

where $p_{T Y_{s}}\left(T Y_{s}\right)$ is the practical histogram distribution of $Y$ and $p_{Y_{s}}\left(Y_{s}\right)$ is the fitting curve according to histogram of complex coefficients $Y$ using (3) while the interscale dependencies are not considered. In other words, when (3) is used to fit the histogram of $Y$, the parameter $K$ calculated in (3) will equal $k_{0}$.

Obviously the most important point of all is that the quantum-inspired probability of noise which is denoted $\cos ^{2}\left(N C_{s m} \times \pi / 2\right)$ adaptively adjusts the shape parameter $K$. If $N C_{s m}$ becomes less, the probability of noise becomes larger; $Y(s, m)$ is very likely to be a noise coefficient of the machinery. The larger the presence probability of noise is and the larger the quantum-inspired adaptive shape parameter $K$ is, the smaller the value of QAWS is, and vice versa. The true signal of the machinery is enhanced and the noise is weakened

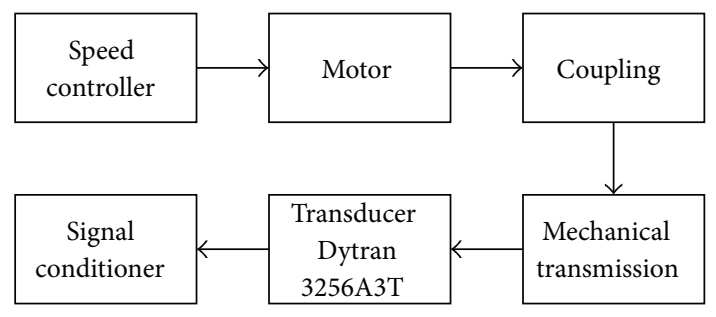

FIGURE 1: The schematic illustration of the mechanical transmission experimental system.

with the QAWS. In QAWS, the nonimportant coefficients are shrank nonlinearly instead of setting to zero, which can not only reduce noise and but also preserve more shape details, such as impulsive feature of fault.

3.3. Procedure of QAWS. The denoising of the mechanical vibration signals employing QAWS can be done in the following way.

(1) Sample a mechanical vibration signal $y$.

(2) Decompose the noisy mechanical vibration signal $y$ by DTCWT.

(3) Estimate the noise variance $\sigma_{n}^{2}$ using (11).

(4) Estimate variance $\sigma^{2}$ using (12).

(5) Estimate parameter $K$ using (19).

(5.1) Calculate the practical histogram distribution of $Y$ and use (3) to fit histogram of $Y$ while the interscale dependencies are not considered.

(5.2) Estimate $k_{0}$ of each level using (20).

(6) Shrink each DTCWT coefficient using QAWS (9).

(7) Apply the inverse DTCWT to the shrank coefficients to obtain the denoised signal.

\section{Application of QAWS for Bearing Signal Denoising}

4.1. Experimental System of Rolling Element Bearing. The vibration signal used in this paper was acquired from a mechanical power-shift steering transmission. The schematic illustration of the mechanical transmission experimental system is shown in Figure 1. The test stand consists of a reliance electric motor, an accelerometer transducer located on tank cover over the fault bearing, and a data recorder with a sampling frequency of $10000 \mathrm{~Hz}$ per channel. The transducer is manufactured by Dytran with product model 3256A3T. The single point fault was introduced to the rolling element bearing at the inner raceway using electrodischarge machining with fault diameter of $3 \mathrm{~mm}$ and fault depth of $2 \mathrm{~mm}$. The shaft rotational speed was $1830 \mathrm{rpm}(30.5 \mathrm{~Hz})$. The time of sampling was 0.15 second. On the basis of the theoretical calculation, the characteristic frequency of inner race fault is $158 \mathrm{~Hz}$. The waveform of the analyzed signal in 


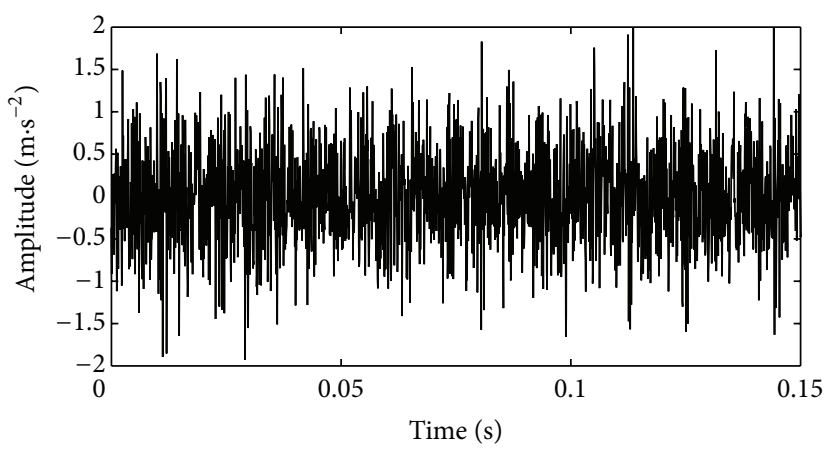

(a)

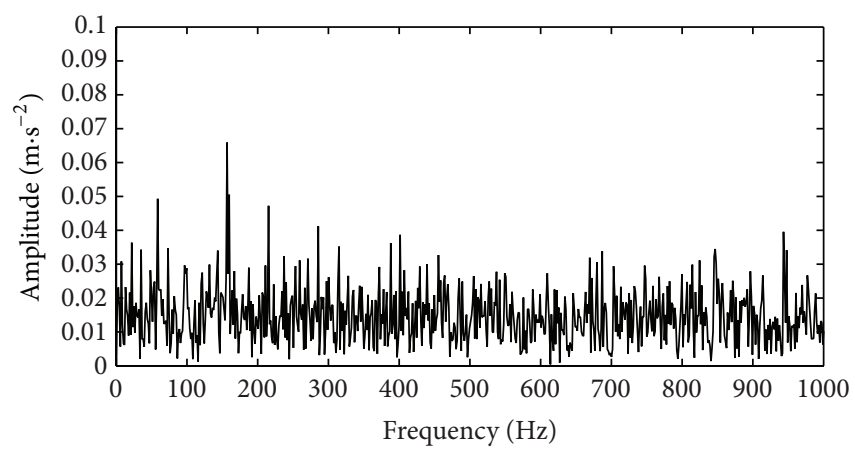

(b)

FIGURE 2: Fault signal with strong noise: (a) waveform in the time domain; (b) frequency spectrum.

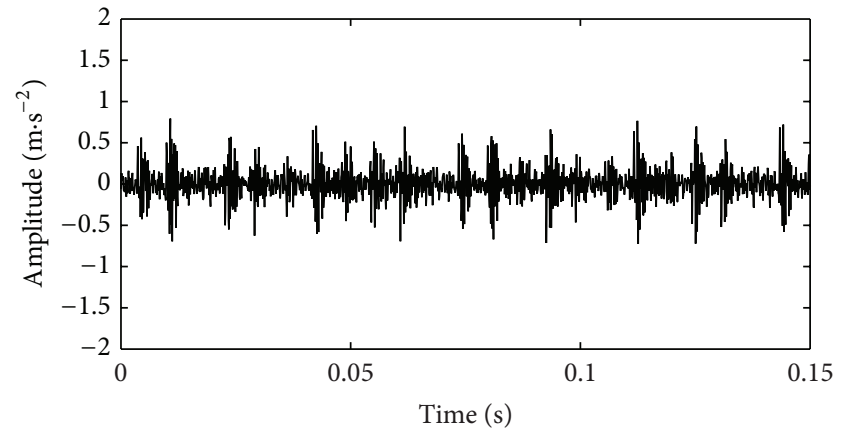

(a)

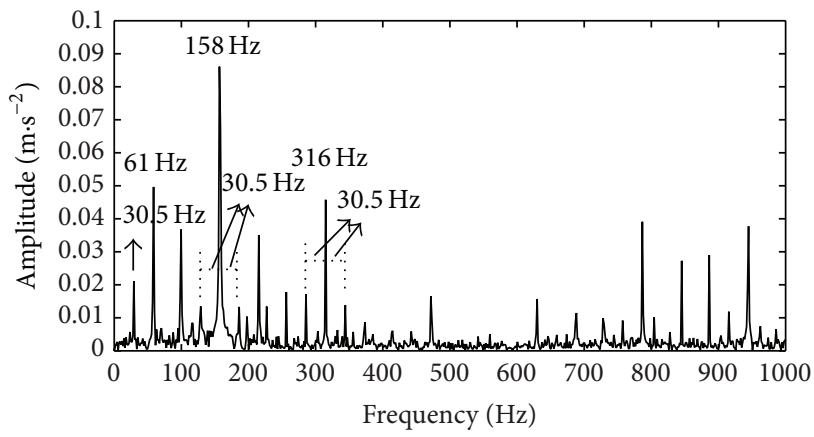

(b)

FIGURE 3: Denoising by QAWS: (a) waveform in the time domain; (b) frequency spectrum.

the time domain and the frequency spectrum are displayed in Figure 2. The defect frequency $158 \mathrm{~Hz}$ and its harmonics are blurred by other useless frequencies, which leads to difficulty in diagnosing the fault in Figure 2(b).

4.2. Applying QAWS for Inner Race Fault Detection. In this section, the vibration signal acquired from a bearing with an inner race defect is analyzed. The presented QAWS scheme is conducted on the signal displayed in Figure 2(a). The waveform and spectrum obtained by using the QAWS is shown in Figure 3. It can be found that the impulsive features generated by fault are clearly extracted in Figure 3(a). The inner race defect characteristic frequency $158 \mathrm{~Hz}$ and its harmonic at $316 \mathrm{~Hz}$ can be identified successfully in Figure 3(b). Furthermore, side frequencies $(158 \pm 30.5 \mathrm{~Hz}, 316 \pm 30.5 \mathrm{~Hz})$ are prominent. The difference between the side frequencies and characteristic frequency or its harmonic frequency is $30.5 \mathrm{~Hz}$ equal to the rotating frequency. In addition, the modulation frequencies $30.5 \mathrm{~Hz}$ (equal to the frequency of rotor rotating) and $61 \mathrm{~Hz}$ (equal to the second harmonic frequency of rotor rotating) are very clear too. For all of these characteristics that accord well with the characteristics of a standard bearing spectrum with inner race fault, we can confirm that there is an inner race fault in the rolling element bearing.

Figures 4 and 5 illustrate the denoising results from the hard and soft thresholding technique. In these two methods, the nonimportant coefficients are set to zero, while the nonimportant coefficients are shrank nonlinearly by QAWS. The characteristic frequency $158 \mathrm{~Hz}$ can be clearly seen in the frequency spectrum. The waveform indicates that a part of impulsive features are extracted. However, the spectrum is still disturbed by other frequency components, which results in difficulty of detecting fault. Characteristic frequency has no sideband and harmonic frequencies. As a whole, the characteristics of the spectrum fail to agree with the characteristics of a bearing with inner race fault. The noise is not depressed effectively by hard and soft thresholding method compared to the QAWS technique.

The QAWS gives the most satisfactory results for the signal: the noise is depressed and the impulsive shape is maintained. Hard and soft thresholding technique can detect the impulsive features but suffer disturbance of noise. It can be found that all three methods can detect the impulsive characteristic frequency $158 \mathrm{~Hz}$. However, the modulation frequency equal to rotating frequency $30.5 \mathrm{~Hz}$ cannot be displayed in the frequency spectrum obtained by hard and 


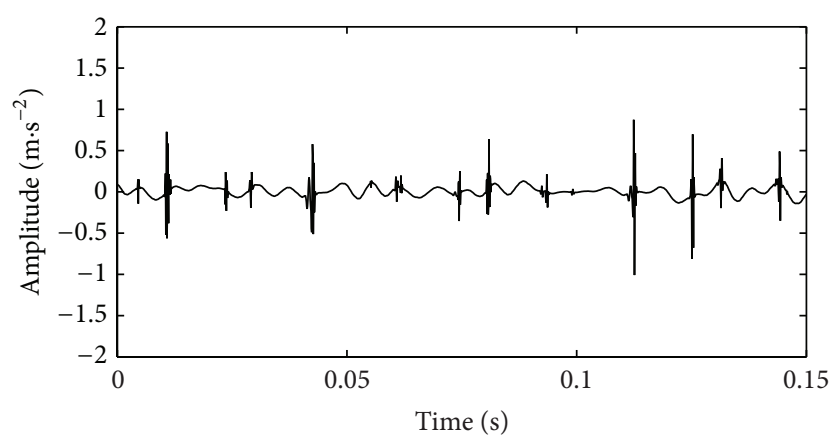

(a)

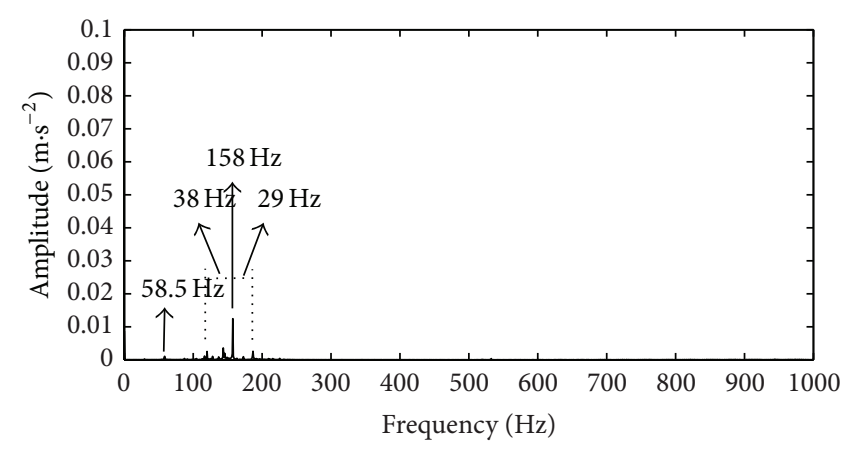

(b)

FIGURE 4: Denoising by soft threshold: (a) waveform in the time domain; (b) frequency spectrum.

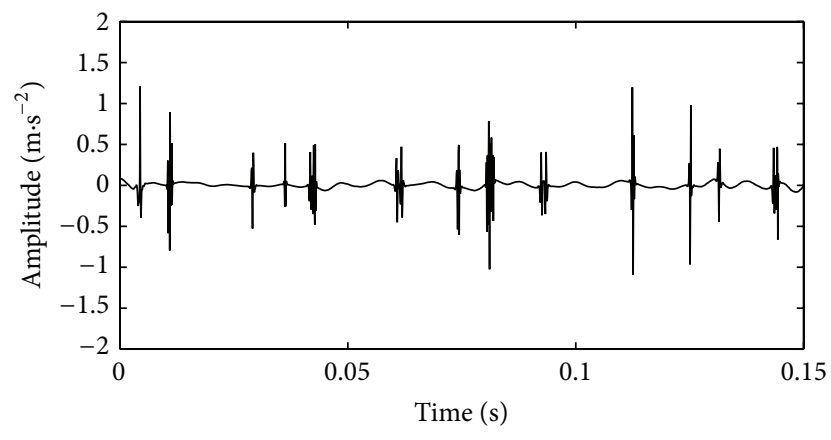

(a)

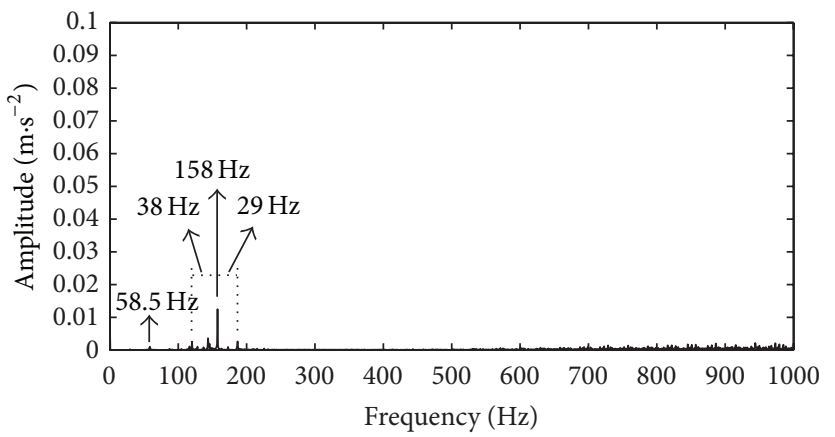

(b)

FIGURE 5: Denoising by hard threshold: (a) waveform in the time domain; (b) frequency spectrum.

soft thresholding. This will affect the analysis of the inner race fault.

\section{Conclusions}

An effective wavelet-domain shrinkage processor that makes use of quantum theory to analyze the interscale dependencies of wavelet subbands is proposed for mechanical vibration signals. The proposed QAWS reduces noise with two innovation points. The first is that an adaptive nonGaussian statistical model of DTCWT coefficients with a tunable parameter is designed, and thus a better practical applicability is achieved. Then, a basic shrinkage function of wavelet coefficient is constructed in a Bayesian framework. The second is that products of the coefficients and their parents are investigated in multiscale subbands, and the quantum-inspired probability of noise is proposed to adjust the tunable parameter in the basic shrinkage function.

Denoising results of rolling element bearings with inner race fault of a mechanical equipment demonstrate that QAWS has a competitive performance to depress noise and preserve details for mechanical vibration signals. Meanwhile, the results also confirm that the proposed QAWS method outperforms the widely used hard and soft thresholding. This algorithm provides a feasible denoising method to improve vibration signals quality. In addition, this research has indicated clearly that the QAWS also can be applied to other machineries.

\section{Nomenclature}

$x: \quad$ A noise free signal

$y: \quad$ A noisy signal

$n: \quad$ White Gaussian noise

$X: \quad$ DTCWT coefficient of $x$

$\widehat{X}: \quad$ Estimation for $X$

$Y: \quad$ DTCWT coefficient of $y$

$N: \quad$ DTCWT coefficient of $n$

$p_{X}(X): \quad$ Statistical model of the DTCWT coefficient $X$

$p_{X \mid Y}(X \mid Y)$ : Condition probability of $X$ given observation $Y$

$p_{N}(N): \quad$ Statistical model of the DTCWT coefficient $N$

$\sigma: \quad$ Standard deviation of $X$

$\widehat{\sigma}: \quad$ Estimation for $\sigma$

$\sigma_{n}: \quad$ Standard deviation of $N$

$\widehat{\sigma_{n}}: \quad$ Estimation for $\sigma_{n}$

$s: \quad$ DCTWT decomposition level

$m: \quad$ The position of DTCWT coefficient at each scale

$Y(s, m)$ : The $m$ th DTCWT coefficient of $Y$ at the sth scale 
$W(s, m)$ : A subwindow centered at wavelet coefficient $Y(s, m)$

$M(s): \quad$ Width of the subwindow $W(s, m)$

$d: \quad$ The finest DTCWT decomposition scale

$\alpha, \beta: \quad$ The probability amplitude

$C_{s m}$ : $\quad$ Product of the coefficient and its parent at $m$ th position of the $s$ th scale level

$N C_{s m}: \quad$ Normalization of $C_{s m}$

$p_{T Y_{s}}\left(T Y_{s}\right)$ : The practical histogram distribution of $Y$

$p_{Y_{s}}\left(Y_{s}\right)$ : The fitting function according to histogram of $Y$

$K: \quad$ Adaptive shape parameter for $p_{X}(X)$

$k_{0}$ : $\quad$ A tunable parameter in $K$ based on mean square error.

\section{Conflict of Interests}

The authors declare that there is no conflict of interests regarding the publication of this paper.

\section{Acknowledgment}

This work was supported by the National Natural Science Foundation of China, under Projects nos. 51205405 and 51305454.

\section{References}

[1] C. Chesneau, J. Fadili, and J.-L. Starck, "Stein block thresholding for image denoising," Applied and Computational Harmonic Analysis, vol. 28, no. 1, pp. 67-88, 2010.

[2] J. N. Taylor, D. E. Makarov, and C. F. Landes, "Denoising single-molecule FRET trajectories with wavelets and Bayesian inference," Biophysical Journal, vol. 98, no. 1, pp. 164-173, 2010.

[3] A. Loza, D. Bull, N. Canagarajah, and A. Achim, "Non-Gaussian model-based fusion of noisy images in the wavelet domain," Computer Vision and Image Understanding, vol. 114, no. 1, pp. 54-65, 2010.

[4] C. C. Liu, T. Y. Sun, S. J. Tsai, Y. Yu, and S. Hsieh, "Heuristic wavelet shrinkage for denoising," Applied Soft Computing Journal, vol. 11, no. 1, pp. 256-264, 2011.

[5] C. M. Chou, "A threshold based wavelet denoising method for hydrological data modelling," Water Resources Management, vol. 25, no. 7, pp. 1809-1830, 2011.

[6] G. Y. Chen and S. E. Qian, "Denoising of hyperspectral imagery using principal component analysis and wavelet shrinkage," IEEE Transactions on Geoscience and Remote Sensing, vol. 49, no. 3, pp. 973-980, 2011.

[7] W. Y. Wang, H. Z. He, and Z. Y. Zi, "Enhancement of signal denoising and multiple fault signatures detecting in rotating machinery using dual-tree complex wavelet transform," Mechanical Systems and Signal Processing, vol. 24, no. 1, pp. 119137, 2010.

[8] R. Shao, W. Hu, and J. Li, "Multi-fault feature extraction and diagnosis of gear transmission system using time-frequency analysis and wavelet threshold denoising based on EMD," Shock and Vibration, vol. 20, no. 2, pp. 341-349, 2013.

[9] C. Cafaro and S. Mancini, "On Grover's search algorithm from a quantum information geometry viewpoint," Physica A:
Statistical Mechanics and its Applications, vol. 391, no. 4, pp. 1610-1625, 2012.

[10] J. Tsai, F. Hsiao, Y. Li, and J. Shen, "A quantum search algorithm for future spacecraft attitude determination," Acta Astronautica, vol. 68, no. 7-8, pp. 1208-1218, 2011.

[11] P. C. Li, K. P. Song, and F. H. Shang, "Double chains quantum genetic algorithm with application to neuro-fuzzy controller design," Advances in Engineering Software, vol. 42, no. 10, pp. 875-886, 2011.

[12] Q. Niu, T. J. Zhou, M. R. Fei et al., "An efficient quantum immune algorithm to minimize mean flow time for hybrid flow shop problems," Mathematics and Computers in Simulation, vol. 84, pp. 1-25, 2012.

[13] R. Vasile, S. Olivares, M. A. Paris, and S. Maniscalco, "Continuous-variable quantum key distribution in nonMarkovian channels," Physical Review A-Atomic, Molecular, and Optical Physics, vol. 83, no. 4, Article ID 042321, 2011.

[14] A. Leverrier and P. Grangier, "Continuous-variable quantumkey-distribution protocols with a non-Gaussian modulation," Physical Review A-Atomic, Molecular, and Optical Physics, vol. 83, no. 4, Article ID 042312, 2011.

[15] X. W. Fu, M. Y. Ding, and C. Cai, "Despeckling of medical ultrasound images based on quantum-inspired adaptivethreshold," Electronics Letters, vol. 46, no. 13, pp. 21-22, 2010.

[16] Y. C. Eldar and A. V. Oppenheim, "Quantum signal processing," IEEE Signal Processing Magazine, vol. 19, no. 6, pp. 12-32, 2002.

[17] D. L. Donoho and J. M. Johnstone, "Ideal spatial adaptation by wavelet shrinkage," Biometrika, vol. 81, no. 3, pp. 425-455, 1994.

[18] U. D. Dwivedi and S. N. Singh, "Enhanced detection of power-quality events using intra and interscale dependencies of wavelet coefficients," IEEE Transactions on Power Delivery, vol. 25, no. 1, pp. 358-366, 2010. 

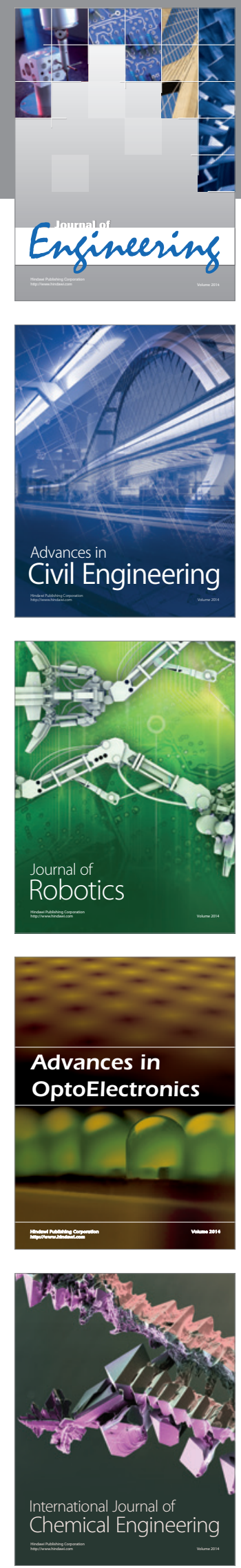

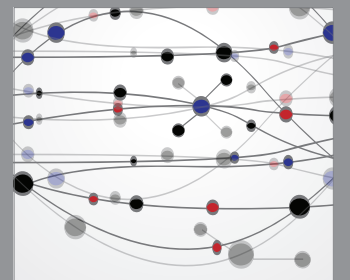

The Scientific World Journal
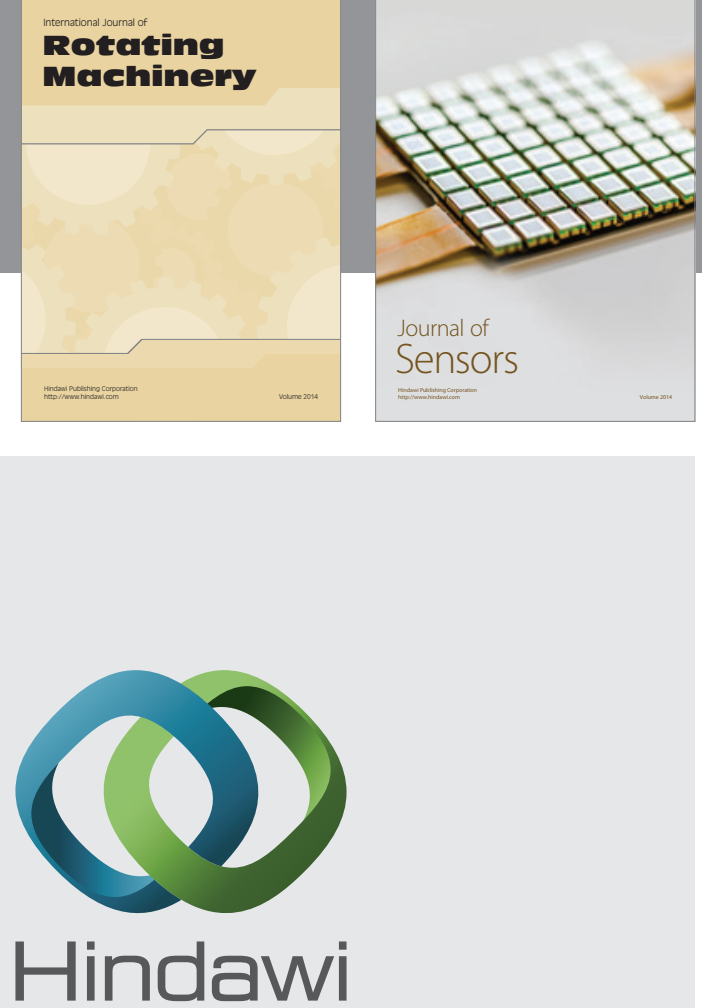

Submit your manuscripts at http://www.hindawi.com
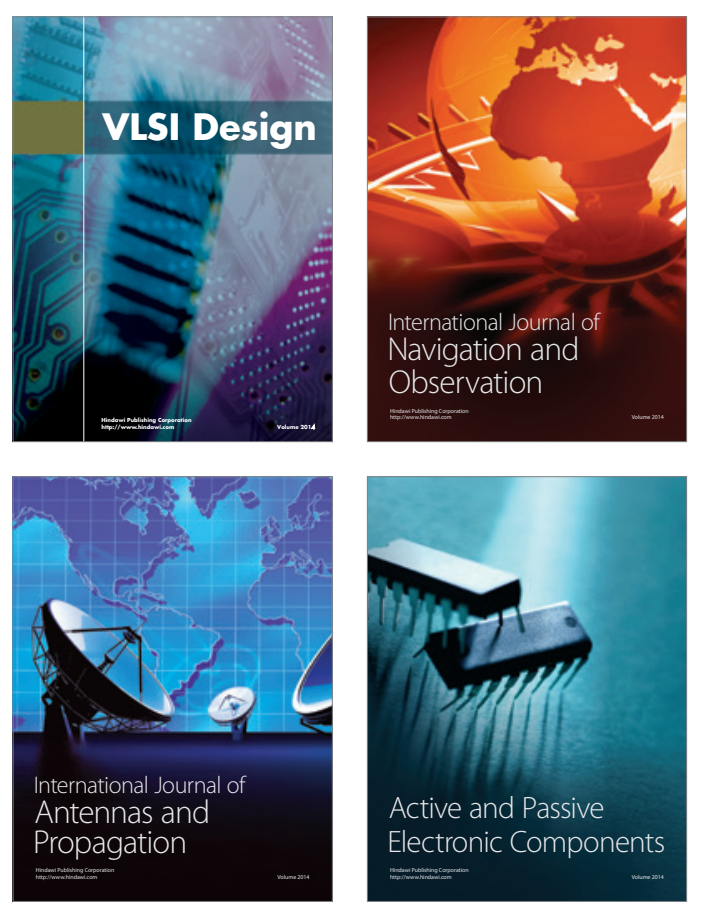
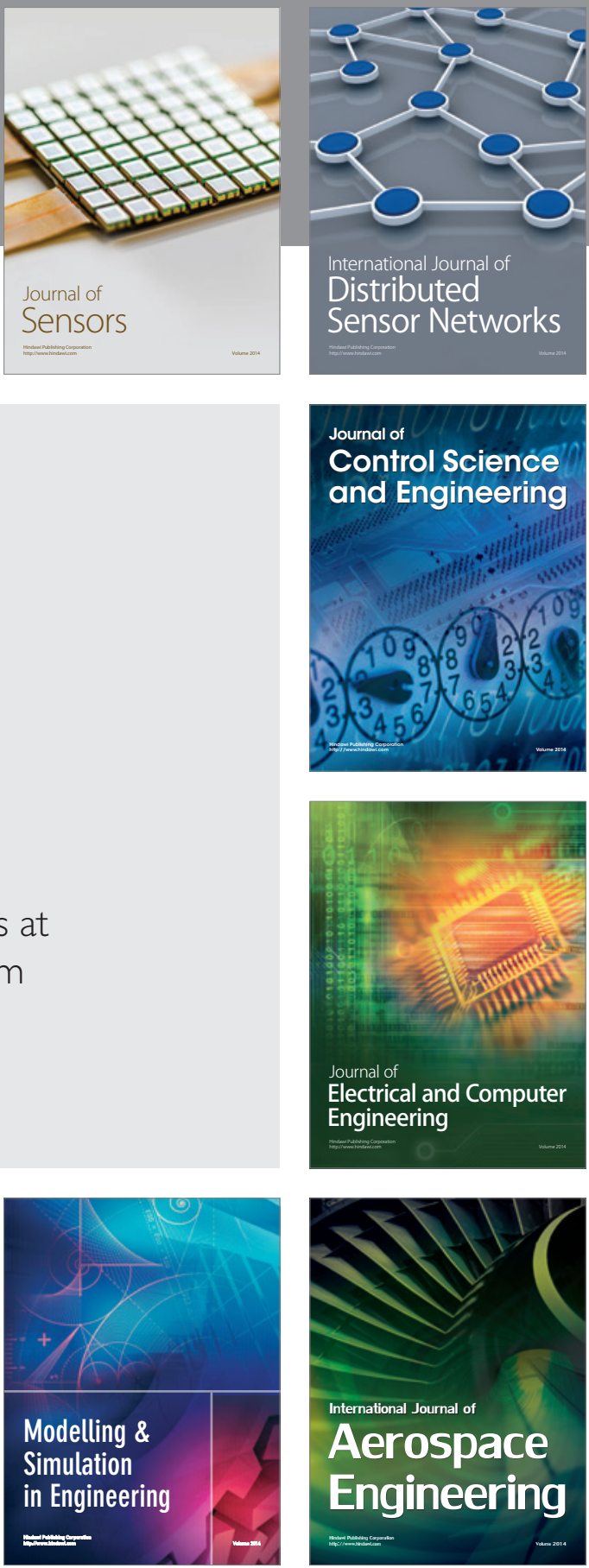

Journal of

Control Science

and Engineering
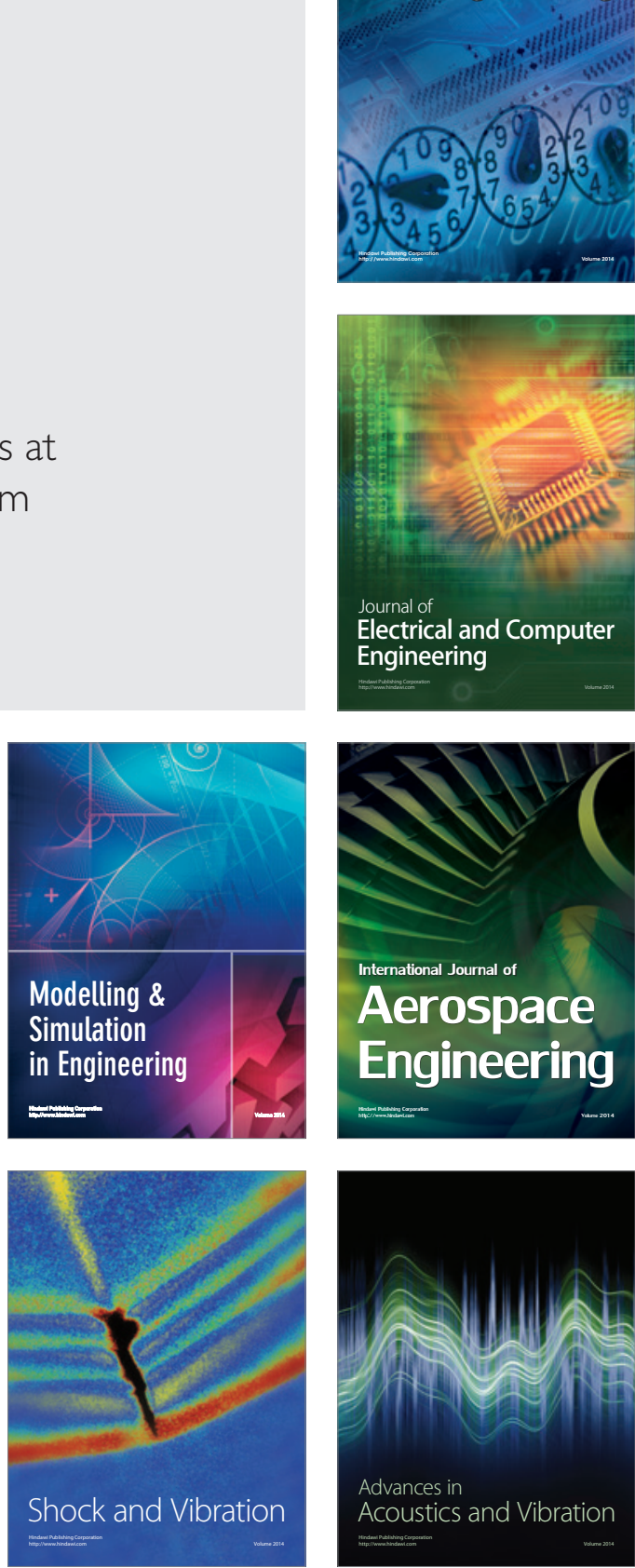